\title{
Fishing for the origins of cancer
}

\author{
Glenn Merlino ${ }^{1,3}$ and Chand Khanna ${ }^{2,4}$ \\ ${ }^{1}$ Laboratory of Cancer Biology and Genetics, National Cancer Institute, National Institutes of Health, Bethesda, Maryland 20892, \\ USA; ${ }^{2}$ Pediatric Oncology Branch, National Cancer Institute, National Institutes of Health, Bethesda, Maryland 20892, USA
}

A hypothesis that is currently in vogue in the field of carcinogenesis suggests that a small, but identifiable population of cells within a tumor provides the "selfrenewal" phenotype of cancer. An extension of this hypothesis is that these self-renewing cells /cancer stem cells) most closely resemble the cell of origin for a given cancer (Reya et al. 2001). Based on this hypothesis, the originating cell of a cancer is increasingly believed to be a primitive cell with the ability to undergo division with high-phenocopy fidelity and retention of multipotency. This intersecting path between developmental biology and cancer is comfortable terrain for those involved in the study of childhood malignancies, particularly sarcomas and lymphoid cancers. The oncogenic events associated with sarcomagenesis and leukemogenesis are in many cases understood and characterized by pathognomonic translocations occurring within identifiable cell lineages (Crans and Sakamoto 2001; Mackall et al. 2002; Helman and Meltzer 2003). This understanding has led to the development and study of several highly relevant animal models of sarcoma and leukemia. Recent technological advances have encouraged extensive interdisciplinary and cross-species analyses of animal models from flies to mice to dogs to humans. These models serve not only in their historical capacity to test hypotheses emerging from the study of human cancer, but to generate new hypotheses that may be overlooked in the study of the human condition alone. This approach is well illustrated in this issue of Genes \& Development by Zon and colleagues (Langenau et al. 2007), who describe the first zebrafish model of rhabdomyosarcoma (RMS), a pediatric malignancy thought to arise from skeletal muscle. Through zebrafish, Langenau et al. (2007) identify an important oncogenic pathway that characterizes a subset of RMS patients as well as a viable candidate for a RMS cell of origin.

\section{Modeling human RMS}

Although not highly prevalent in the human population, RMS nevertheless represents the most common soft-tissue sarcoma of childhood. Patients diagnosed with advanced-stage RMS face a grave prognosis. Like other pe-

Correspondence.

${ }^{3}$ E-MAIL gmerlino@helix.nih.gov; FAX (301) 480-7618.

${ }^{4}$ E-MAIL khannac@mail.nih.gov; FAX (301) 443-1838.

Article is online at http://www.genesdev.org/cgi/doi/10.1101/gad.1563707. diatric sarcomas, RMS can be broadly divided into those with simple karyotypic defects (i.e., reciprocal chromosomal translocations that encode chimeric fusion proteins) and those with more complex karyotypic defects, but lacking characteristic chromosome markers. The current classification of RMS into two major and several minor subtypes is supported by histopathological criteria, the presence of signature chromosomal markers, and most recently by genome-wide expression profiling (Helman and Meltzer 2003; Mercado and Barr 2007). Embryonal RMS (ERMS), the most common and more treatable subtype, typically occurs in infants and young children. There is no distinct molecular event that characterizes ERMS, but they tend to demonstrate loss of allelic heterozygosity (i.e., at chromosome $11 \mathrm{p} 15$ ) and severe genomic instability. In contrast, alveolar RMS (ARMS) are highly aggressive tumors of adolescents and young adults, with $70 \%$ classified based on translocations involving the genes encoding the forkhead transcription factor (FKHR, or FOXO1A), and either PAX3 or PAX7. These translocation-positive ARMS possess a distinctive homogeneous molecular signature, whereas the $30 \%$ of ARMS that lack PAX-FKHR fusion proteins possess no discernible signature (Wachtel et al. 2004). The genomic distinction between RMS subtypes enables unambiguous classification, and may uncover subtype-specific treatment targets. To fully realize the potential of modern human genomics, relevant animal models will be required to provide a platform for generating and validating novel hypotheses and to identify and test therapeutic targets and agents.

Just a few short years ago there were virtually no animal models for any subtypes of human RMS. Today, a number of useful mouse models of RMS have been reported that have proven to be highly relevant at the molecular level. With respect to ARMS, great efforts have been expended to express the PAX3-FKHR fusion protein in an appropriate target cell in the mouse, but most have resulted in variable expression and embryonic lethality (Mercado and Barr 2007). However, Keller et al. (2004) succeeded in producing a mouse model of ARMS through conditional activation of $\mathrm{Pax} 3-\mathrm{Fkhr}$ by targeting Cre expression to Myf6-expressing skeletal muscle cells; simultaneous inactivation of either Ink4a/Arf or Tp53 accelerated ARMS formation. Sharp et al. (2002) created a model in which the combination of deregulation of the receptor tyrosine kinase (RTK) MET and disruption of 
Ink4a/Arf rapidly induced highly aggressive ERMS; deficiency in p53 was found to be as effective as loss of Ink4a/Arf in this model (T. Ichikawa and G. Merlino, unpubl.). Similarly, expression of activated HER-2/neu induced RMS in p53-deficient mice (Nanni et al. 2003). Loss of p53 was also found to cooperate with FOS (Fleischmann et al. 2003) and activated KRAS (Tsumura et al. 2006) to induce RMS; in the latter, lesions developed resembling pleomorphic RMS.

When taken together, it is tempting to conclude from these model systems that disruption along the ARF-p53 axis is central to the development of RMS, and can operate in conjunction with deregulated RTK signaling, particularly signaling through RAS-associated pathways. Extensive studies of human RMS tissues have substantiated the former concept, where TP53 mutations are fairly common (Merlino and Helman 1999; Helman and Meltzer 2003). However, mutations in RAS genes in human RMS are actually relatively rare (reports ranging between $4 \%$ and 35\%) (Stratton et al. 1989; Chen et al. 2006). Enter the zebrafish.

\section{Zebrafish genomics and RMS pathways}

Zebrafish, the striped teleost Danio rerio, possess myriad biological properties that have made them a workhorse for the study of embryogenesis and developmental pathways for over a quarter of a century. Zebrafish mating results in large numbers of optically clear, rapidly developing embryos, suitable for unimpeded observation and sophisticated ex utero manipulation. Moreover, zebrafish are ideal models for both forward and reverse genetic analyses, enabled by the currently advanced understanding of zebrafish genomics. These same properties also make the zebrafish a powerful model system for studying human cancer (Stern and Zon 2003; Berghmans et al. 2005). Genetic screens can be performed following exposure to carcinogens (i.e., ethylnitrosourea) or insertional mutagenesis, and transgenic zebrafish models have been generated for a variety of human carcinomas, sarcomas, and hematological tumors. Tumor suppressor gene disruption can be achieved by a screening strategy called targeting-induced local lesions in genomes (TILLING) and by Cre/loxP-mediated site-directed recombination (Langenau et al. 2005; Pan et al. 2005); targeted expression of oncogenes is now fairly routine. Furthermore, validation of genotype-phenotype relations in zebrafish has been facilitated by the relatively simple knockdown of gene expression using morpholino constructs. The rapid, well-characterized vascular development in zebrafish has also been used to argue for their inclusion in high-throughput screens for anti-cancer agents that disrupt angiogenesis or vasculogenesis.

Working with zebrafish, Langenau et al. (2007) discovered that a transgene in which expression of human activated KRAS (kRASG12D) was driven by a rag2 promoter rapidly induced tumors that appeared to be skeletal muscle in origin, based on the presence of multinucleated striated muscle fibers and a battery of diagnostic markers (i.e., desmin, myod, met, myf5, m- cadherin), but very few lymphoid tumors. Gene-expression profiling was used to validate that the zebrafish tumors were consistent with a human RMS. Gene set enrichment analysis (GSEA), already successful in classifying human, mouse, and even zebrafish cancer (Ramaswamy et al. 2001; Sweet-Cordero et al. 2005; Lam et al. 2006), was used to determine whether a gene set derived from their zebrafish model (tumor vs. normal muscle) was enriched in human data sets of a variety of tumors vs. their corresponding normal tissues. Notably, the gene sets up-regulated in the zebrafish tumors (but interestingly not the down-regulated sets) were significantly associated with ERMS, but not ARMS, renal cell carcinoma, colon, lung, or prostate adenocarcinoma data sets, thereby validating this transgenic zebrafish as a model for human ERMS and providing new candidate markers for its diagnosis.

Interestingly, the up-regulated zebrafish gene set was also significantly associated with that of human pancreatic adenocarcinoma, $90 \%$ of which harbor activating KRAS mutations, as well as kRASG12D-induced mouse lung adenocarcinoma (Iacobuzio-Donahue et al. 2003; Sweet-Cordero et al. 2005), suggesting that these tumors share a common RAS signature-a notion that was confirmed by comparison to human cells infected with activated RAS. These cross-species comparative genomic data uncovered the existence of a RAS signature in human ERMS that typically do not harbor mutated RAS, and for which no previous RAS signature had been recognized. It is thought that the incorporation of the relatively homogeneous tumor samples derived from genetically engineered mouse, and now fish models, provides a filter that can facilitate the detection of otherwise hidden signatures in a population of histologically similar, but heterogeneous patient tumors.

\section{Zebrafish and the origin of RMS}

The importance of the role of activated RAS, or an equivalent signature, in RMS as well as other cancer types is highly dependent on the cell type to which its expression is directed (Brown et al. 1998). For example, myoblasts are long known to be differentiation defective in the presence of activated RAS (Schneider and Olson 1988). Notably, in a mouse lung adenocarcinoma model, expression of activated KRAS is fully compatible with self-renewal, enabling expansion of the bronchioalveolar stem cell population (Kim et al. 2005). Langenau et al. (2007) chose to direct expression of the kRASG12D transgene in zebrafish using a rag2 promoter, normally expected to target immature $\mathrm{B}$ and $\mathrm{T}$ cells. Although a seemingly odd choice to initiate a study of skeletal muscle cancer, its utility was demonstrated when EGFP and dsRED2 fluorescent markers expressed via the same rag2 promoter were shown to aberrantly target a mononuclear population within the zebrafish musculature that included satellite cells and differentiating myoblasts, but not multinucleated terminally differentiating muscle fibers.

Given the impressive induction of zebrafish ERMS through ectopic expression of kRASG12D, a critical is- 
sue became the identity of the tumor-initiating cell targeted by the rag2 promoter. Langenau et al. (2007) addressed this question by exploiting a novel targeting and labeling strategy to allow the identification and molecular characterization of distinct pools of tumor cells from zebrafish ERMS (Fig. 1A). By coinjecting both rag2dsRED2 and rag2-kRASG12D constructs into $\alpha$-actinGFP transgenic zebrafish embryos, they were able to differentially label RMS cells based on their differentiation status: rag2 promoter-directed red fluorescence would mark mononuclear myogenic precursors $\left(\mathrm{R}^{+}\right)$, while $\alpha$-actin promoter-driven green fluorescence would label more mature muscle cells $\left(\mathrm{G}^{+}\right) . \mathrm{R}^{+} \mathrm{G}^{+}$cells in RMS tumors represented cells of intermediate muscle differentiation, while double-negative cells were positive for blood cell markers. Using serial transplantation and limiting-dilution transplantation, functional hallmarks for cancer stem cell behavior, Langenau et al. (2007) found greater stem-like potential among the less-well-differentiated myogenic population $\left(\mathrm{R}^{+}\right)$, likely representing the target cells for KRAS transformation (Fig. 1B). Based on microarray analysis, Langenau et al. (2007) concluded that the $\mathrm{R}^{+}$population found in zebrafish ERMS was molecularly similar to activated satellite cells and shared pathways involved in normal satellite cell self-renewal. This $\mathrm{R}^{+}$population was characterized by up-regulation of Notch1, Id2, and Gli3, consistent with alterations in Notch and Wnt signaling, both important in stem cell function (Fig. 1C). Taken together, the data suggest that activated KRAS can promote self-renewal while simultaneously inhibiting differentiation of the RMS cell of origin.

The zebrafish model described by Langenau et al. (2007) contributes to an ongoing discussion concerning the RMS cell of origin. Two candidate target cells for RMS are thought to be the committed muscle stem cell (i.e., the satellite cell) and the multipotent mesenchymal stem cell (MSC). To date, the issue of cell of origin has not been resolved, although animal models are beginning to provide new insight. It is likely that either of these cell types, and indeed other cells in various stages of lineage differentiation, can give rise to RMS, depending on a number of critical factors: the nature of the initiating oncogenic event, the environment, and a quality of the cell that could be called permissivity.

The permissive state of a target cell may be defined through its capacity to tolerate and respond to oncogenic events, thereby determining its potential to evolve into a tumor. A cell's permissivity can change, influenced by its developmental state, including its ability to self-renew and genetic determinants, including predisposing mutations (Barr 1998). The permissive cell may be more easily studied in lymphoid cancers and certain sarcomas, where many simple oncogenic reciprocal chromosomal translocations have been defined as sufficient for transformation (Crans and Sakamoto 2001; Helman and Meltzer 2003; Mercado and Barr 2007). Sarcoma-specific chimeric fusion proteins are thought to act as explosively powerful transcription factors, offering transformed cells advantages in growth, motility, and/or apoptotic avoidance (Mackall et al. 2002; Helman and Meltzer 2003).

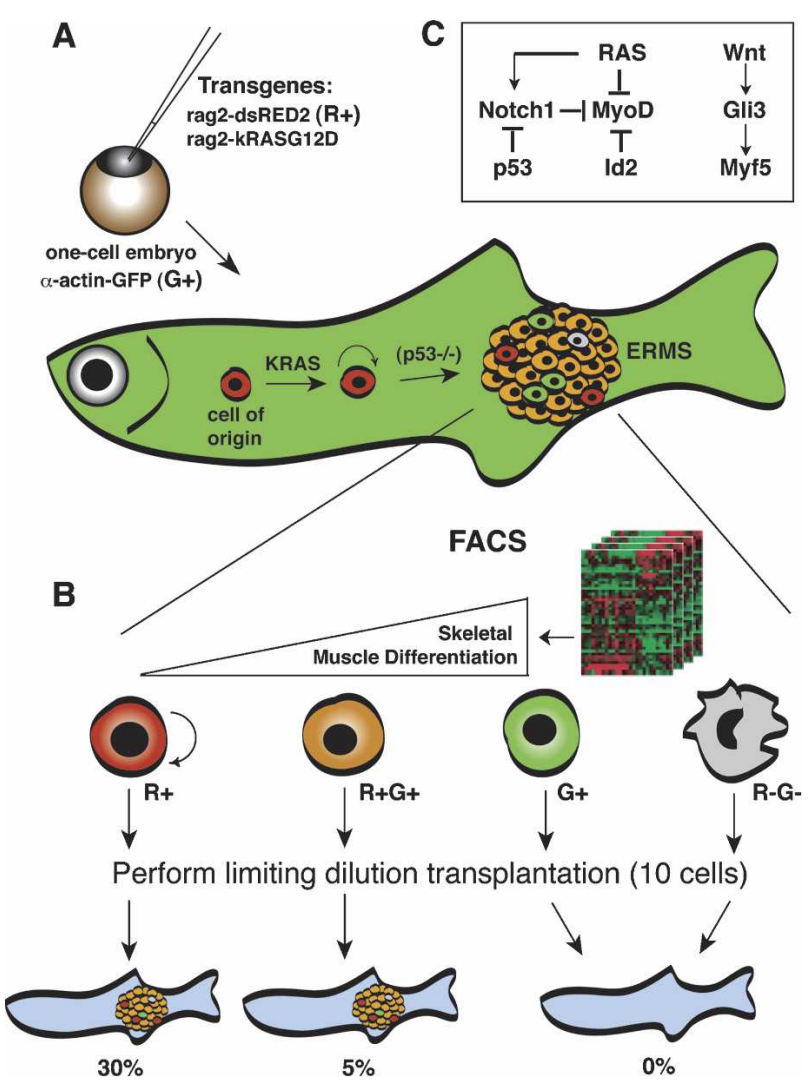

Figure 1. Zebrafish help unravel the cellular origins of ERMS. (A) Cosegregating transgenes targeting expression of dsRED2 and oncogenic kRASG12D to satellite cells and early myogenic progenitors $\left(\mathrm{R}^{+}\right)$are introduced into transgenic zebrafish embryos expressing GFP in more mature skeletal muscle $\left(\mathrm{G}^{+}\right)$. Although activated KRAS is sufficient to rapidly induce tumors resembling ERMS, rhabdomyosarcomagenesis can be enhanced in the absence of p53. (B) Arising zebrafish ERMS consist of $\mathrm{R}^{+}$ and $\mathrm{G}^{+}$cells, as well as double-positive $\left(\mathrm{R}^{+} \mathrm{G}^{+}\right)$and double-negative $\left(\mathrm{R}^{-} \mathrm{G}^{-}\right)$cells, which can be isolated in relatively pure populations by FACS and subjected to array-based expression profiling. $\mathrm{R}^{+}, \mathrm{R}^{+} \mathrm{G}^{+}$, and $\mathrm{G}^{+}$populations were found to represent myogenic cells in increasing states of differentiation, while the $\mathrm{R}^{-} \mathrm{G}^{-}$ cells were blood cells expressing macrophage markers. ERMS could be serially transplanted, and the purified $\mathrm{R}^{+}$population was especially proficient at regenerating entire tumors, even when introduced into recipient fish using as few as 10 cells (percentage shown at bottom is based on the sum of all dilution transplantation experiments presented). $\mathrm{R}^{+}$cells possessing these stem-like properties were molecularly similar to activated satellite cells. $(C)$ Hypothetical scheme highlighting pathways that might facilitate rhabdomyosarcomagenesis. Oncogenic RAS is long known to block skeletal myogenesis and induce satellite cell proliferation and can also activate signaling by Notch1 (Weijzen et al. 2002), highly expressed in the $\mathrm{R}^{+}$population. $\mathrm{R}^{+}$cells also overexpress Id2, an inhibitor of MyoD, as well as Gli3, which is downstream from Wnt. Notch and Wnt pathways are heavily implicated in stem cell maintenance and self-renewal in hematopoietic and other lineages (Reya et al. 2001). It is also worth noting that p53 has been shown to suppress self-renewal in neural stem cells (Meletis et al. 2006). Connectors are meant to indicate functional associations, not necessarily direct interactions. 
In sarcomas harboring such simple but potent oncogenic translocations, an interesting experimental observation supports the concept of the permissive cell. Ectopic expression of these oncogenic gene fusion proteins can be deleterious or lethal to many cell types, which indicates a unique tolerance or permissivity among the true originating cells of translocation-bearing sarcomas (Deneen and Denny 2001; Lagutina et al. 2002; Lessnick et al. 2002). To be effective, the permissive cell must be programmed to accept and survive the activation of potent new signaling pathways and the resultant changes in phenotype (multipotency), and be able to respond by undergoing self-renewal and expansion. Such attributes could be used to describe primitive cells with stem-like properties. Based on this line of reasoning, an intriguing possibility arises that the MSC represents a permissive or originating cell for many sarcomas (Mackall et al. 2002; Helman and Meltzer 2003; Tolar et al. 2007). The established pluripotent capacity of MSCs to differentiate into all of the known lineages of adult mesenchymal cells supports this emerging hypothesis. Ultimate commitment to a cellular lineage could occur as a consequence of the specific initiating oncogenic event (Barr 1998) and the differentiation pathways through which it interacts, as well as by environmental cues received by the newly transformed cell.

We posit that highly potent oncogenic events such as the creation of sarcoma-specific signature chromosomal translocations will normally only be tolerated in globally permissive tumor-initiating cells, consistent with the MSC phenotype. In support of this, primary mesenchymal progenitor cells have been shown to uniquely tolerate expression of the FUS-CHOP and EWS-FLI-1 fusion proteins, and respond by forming myxoid liposarcomas and Ewing's sarcoma, respectively (Castillero-Trejo et al. 2005; Riggi et al. 2005, 2006). However, more differentiated cells already committed to a lineage (i.e., myogenic stem cells or committed satellite cells in skeletal muscle) are more restricted in their permissivity, and may only tolerate weaker oncogenic events, such as RAS activation. More committed cells can dedifferentiate and enhance their stem-like properties, including the capacity to self-renew and then develop into the more complex karyotypic sarcomas lacking characteristic chromosomal translocations (i.e., ERMS) through the acquisition of additional step-wise genetic events, so-called "second hits." In the zebrafish ERMS model, KRAS would serve as the initiating event for the satellite cell, and loss of p53 function might represent a second hit, as tumor incidence was enhanced in p53-deficient zebrafish (Langenau et al. 2007).

Implicit in this hypothesis is the possibility that the cell of origin for simple karyotype RMS may normally be distinct from that giving rise to complex karyotype RMS; however, there is no clear demarcation between these two scenarios. The line blurs if more differentiated cell types "learn" to tolerate and respond to the presence of PAX3-FKHR fusion proteins by transitioning to a more permissive state as a consequence of specific environmental, epigenetic, and/or genetic alterations, such as loss of INK4A/ARF or p53 (Deneen and Denny 2001; Lessnick et al. 2002). This would help explain why Keller et al. (2004) succeeded in coaxing ARMS from a more differentiated myogenic cell in conditional Pax3-Fkhr mice deficient in either p53 or Ink4a/Arf. Further illustrating the complexity of this issue, Haldar et al. (2007) recently reported that synovial sarcoma, a cancer of unclear cellular origin, could be produced in mice by targeting only its defining translocation-derived chimeric SYT-SSX fusion protein to immature myoblasts. Finally, it is worth noting that the nature of the cell of origin is likely to influence the age of onset of RMS in patients through a variety of factors, including the number of second hits required to generate the RMS and the size of the available pool of target cells (i.e., MSCs or myogenic stem cells).

\section{Looking downstream from the zebrafish}

The aforementioned hypothetical relationship between cells in various states of myogenic differentiation and the oncogenic signals that initiate RMS remain to be experimentally validated. The zebrafish described by Langenau et al. (2007) constitutes a high-throughput model in which to do just that. The results of this experimental approach will provide novel insights into cancer stem cell biology, and perhaps significantly impact the course of future cancer treatment.

The therapeutic ramifications of the cancer stem cell hypothesis argue that effective and long-term treatments for cancer must ultimately target the self-renewing population of cancer cells. Current cancer therapies may fail to target this largely quiescent but transiently selfrenewing population of cells. Accordingly, after initial attempts at therapy, the recurrence and progression of cancer is defined by survival and repopulation of the tumor by the cancer stem cell. Efforts to define and characterize these cancer stem cell populations have been problematic, but will be improved through an understanding of the cell of origin for each cancer. A zebrafish model has now been developed that will greatly enhance our understanding of the origin of ERMS. Prospects are further brightened by the fact that zebrafish are well suited to genetic screens and highly amenable to highthroughput chemical screens, facilitating the future discovery of RMS-suppressing drugs. By using experimental models like the zebrafish to develop more effective drugs for the treatment of RMS, we can make sure that the big one doesn't get away.

\section{Acknowledgments}

We thank Drs. Lee Helman, Paul Meltzer, and Lalage Wakefield for critical review of this manuscript.

\section{References}

Barr, F.G. 1998. Translocations, cancer and the puzzle of specificity. Nat. Genet. 19: 121-124.

Berghmans, S., Jette, C., Langenau, D., Hsu, K., Stewart, R., Look, T., and Kanki, J.P. 2005. Making waves in cancer research: New models in the zebrafish. Biotechniques 39: 227-237.

Brown, K., Strathdee, D., Bryson, S., Lambie, W., and Balmain, 
A. 1998. The malignant capacity of skin tumours induced by expression of a mutant H-ras transgene depends on the cell type targeted. Curr. Biol. 8: 516-524.

Castillero-Trejo, Y., Eliazer, S., Xiang, L., Richardson, J.A., and Ilaria, R.L. 2005. Expression of the EWS/FLI-1 oncogene in murine primary bone-derived cells results in EWS/FLI-1-dependent, Ewing sarcoma-like tumors. Cancer Res. 65: 8698-8705.

Chen, Y., Takita, J., Hiwatari, M., Igarashi, T., Hanada, R., Kikuchi, A., Hongo, T., Taki, T., Ogasawara, M., Shimada, A., et al. 2006. Mutations of the PTPN11 and RAS genes in rhabdomyosarcoma and pediatric hematological malignancies. Genes Chromosomes Cancer 45: 583-591.

Crans, H.N. and Sakamoto, K.M. 2001. Transcription factors and translocations in lymphoid and myeloid leukemia. Leukemia 15: 313-331.

Deneen, B. and Denny, C.T. 2001. Loss of p16 pathways stabilizes EWS/FLI1 expression and complements EWS/FLI1 mediated transformation. Oncogene 20: 6731-6741.

Fleischmann, A., Jochum, W., Eferl, R., Witowsky, J., and Wagner, E.F. 2003. Rhabdomyosarcoma development in mice lacking Trp53 and Fos: Tumor suppression by the Fos protooncogene. Cancer Cell 4: 477-482.

Haldar, M., Hancock, J.D., Coffin, C.M., Lessnick, S.L., and Capecchi, M.R. 2007. A conditional mouse model of synovial sarcoma: Insights into a myogenic origin. Cancer Cell 11: 375-388.

Helman, L.J. and Meltzer, P. 2003. Mechanisms of sarcoma development. Nat. Rev. Cancer 3: 685-694.

Iacobuzio-Donahue, C.A., Maitra, A., Olsen, M., Lowe, A.W., van Heek, N.T., Rosty, C., Walter, K., Sato, N., Parker, A., Ashfaq, R., et al. 2003. Exploration of global gene expression patterns in pancreatic adenocarcinoma using cDNA microarrays. Am. J. Pathol. 162: 1151-1162.

Keller, C., Arenkiel, B.R., Coffin, C.M., El-Bardeesy, N., DePinho, R.A., and Capecchi, M.R. 2004. Alveolar rhabdomyosarcomas in conditional Pax3:Fkhr mice: Cooperativity of Ink4a/ARF and Trp53 loss of function. Genes \& Dev. 18: 2614-2626.

Kim, C.F., Jackson, E.L., Woolfenden, A.E., Lawrence, S., Babar, I., Vogel, S., Crowley, D., Bronson, R.T., and Jacks, T. 2005. Identification of bronchioalveolar stem cells in normal lung and lung cancer. Cell 121: 823-835.

Lagutina, I., Conway, S.J., Sublett, J., and Grosveld, G.C. 2002. Pax3-FKHR knock-in mice show developmental aberrations but do not develop tumors. Mol. Cell. Biol. 22: 7204-7216.

Lam, S.H., Wu, Y.L., Vega, V.B., Miller, L.D., Spitsbergen, J., Tong, Y., Zhan, H., Govindarajan, K.R., Lee, S., Mathavan, S., et al. 2006. Conservation of gene expression signatures between zebrafish and human liver tumors and tumor progression. Nat. Biotechnol. 24: 73-75.

Langenau, D.M., Feng, H., Berghmans, S., Kanki, J.P., Kutok, J.L., and Look, A.T. 2005. Cre/lox-regulated transgenic zebrafish model with conditional myc-induced $T$ cell acute lymphoblastic leukemia. Proc. Natl. Acad. Sci. 102: 6068-6073.

Langenau, D.M., Keefe, M.D., Storer, N.Y., Guyon, J.R., Kutok, J.L., Le, X., Goessling, W., Neuberg, D.S., Kunkel, L.M., and Zon, L.I. 2007. Effects of RAS on the genesis of embryonal rhabdomyosarcoma. Genes \& Dev. (this issue) doi: 10.1101/ gad.1545007.

Lessnick, S.L., Dacwag, C.S., and Golub, T.R. 2002. The Ewing's sarcoma oncoprotein EWS/FLI induces a p53-dependent growth arrest in primary human fibroblasts. Cancer Cell 1: 393-401.

Mackall, C.L., Meltzer, P.S., and Helman, L.J. 2002. Focus on sarcomas. Cancer Cell 2: 175-178.

Meletis, K., Wirta, V., Hede, S.M., Nister, M., Lundeberg, J., and Frisen, J. 2006. p53 suppresses the self-renewal of adult neu- ral stem cells. Development 133: 363-369.

Mercado, G.E. and Barr, F.G. 2007. Fusions involving PAX and FOX genes in the molecular pathogenesis of alveolar rhabdomyosarcoma: Recent advances. Curr. Mol. Med. 7: 47-61.

Merlino, G. and Helman, L.J. 1999. RhabdomyosarcomaWorking out the pathways. Oncogene 18: 5340-5348.

Nanni, P., Nicoletti, G., De Giovanni, C., Croci, S., Astolfi, A., Landuzzi, L., Di Carlo, E., Iezzi, M., Musiani, P., and Lollini, P.L. 2003. Development of rhabdomyosarcoma in HER-2/ neu transgenic p53 mutant mice. Cancer Res. 63: 2728-2732.

Pan, X., Wan, H., Chia, W., Tong, Y., and Gong, Z. 2005. Demonstration of site-directed recombination in transgenic zebrafish using the Cre/loxP system. Transgenic Res. 14: 217-223.

Ramaswamy, S., Tamayo, P., Rifkin, R., Mukherjee, S., Yeang, C.H., Angelo, M., Ladd, C., Reich, M., Latulippe, E., Mesirov, J.P., et al. 2001. Multiclass cancer diagnosis using tumor gene expression signatures. Proc. Natl. Acad. Sci. 98: 15149-15154.

Reya, T., Morrison, S.J., Clarke, M.F., and Weissman, I.L. 2001. Stem cells, cancer, and cancer stem cells. Nature 414: 105-111.

Riggi, N., Cironi, L., Provero, P., Suvà, M.L., Kaloulis, K., Garcia-Echeverria, C., Hoffmann, F., Trumpp, A., and Stamenkovic, I. 2005. Development of Ewing's sarcoma from primary bone marrow-derived mesenchymal progenitor cells. Cancer Res. 65: 11459-11468.

Riggi, N., Cironi, L., Provero, P., Suvà, M., Stehle, J., Baumer, K., Guillou, L., and Stamenkovic, I. 2006. Expression of the FUS-CHOP fusion protein in primary mesenchymal progenitor cells gives rise to a model of myxoid liposarcoma. Cancer Res. 66: 7016-7023.

Schneider, M.D. and Olson, E.N. 1988. Control of myogenic differentiation by cellular oncogenes. Mol. Neurobiol. 2: 1-39.

Sharp, R., Recio, J.A., Jhappan, C., Otsuka, T., Liu, S., Yu, Y., Liu, W., Anver, M., Navid, F., Helman, L.J., et al. 2002. Synergism between INK4a/ARF inactivation and aberrant HGF/SF signaling in rhabdomyosarcomagenesis. Nat. Med. 8: 1276-1280.

Stern, H.M. and Zon, L.I. 2003. Cancer genetics and drug discovery in the zebrafish. Nat. Rev. Cancer 3: 533-539.

Stratton, M.R., Fisher, C., Gusterson, B.A., and Cooper, C.S. 1989. Detection of point mutations in N-ras and K-ras genes of human embryonal rhabdomyosarcomas using oligonucleotide probes and the polymerase chain reaction. Cancer Res. 49: 6324-6327.

Sweet-Cordero, A., Mukherjee, S., Subramanian, A., You, H., Roix, J.J., Ladd-Acosta, C., Mesirov, J., Golub, T.R., and Jacks, T. 2005. An oncogenic KRAS2 expression signature identified by cross-species gene-expression analysis. Nat. Genet. 37: 48-55.

Tolar, J., Nauta, A.J., Osborn, M.J., Panoskaltsis Mortari, A., McElmurry, R.T., Bell, S., Xia, L., Zhou, N., Riddle, M., Schroeder, T.M., et al. 2007. Sarcoma derived from cultured mesenchymal stem cells. Stem Cells 25: 371-379.

Tsumura, H., Yoshida, T., Saito, H., Imanaka-Yoshida, K., and Suzuki, N. 2006. Cooperation of oncogenic K-ras and p53 deficiency in pleomorphic rhabdomyosarcoma development in adult mice. Oncogene 25: 7673-7679.

Wachtel, M., Dettling, M., Koscielniak, E., Stegmaier, S., Treuner, J., Simon-Klingenstein, K., Buhlmann, P., Niggli, F.K., and Schafer, B.W. 2004. Gene expression signatures identify rhabdomyosarcoma subtypes and detect a novel $\mathrm{t}(2 ; 2)(\mathrm{q} 35 ; \mathrm{p} 23)$ translocation fusing PAX3 to NCOA1. Cancer Res. 64: 5539-5545.

Weijzen, S., Rizzo, P., Braid, M., Vaishnav, R., Jonkheer, S.M., Zlobin, A., Osborne, B.A., Gottipati, S., Aster, J.C., Hahn, W.C., et al. 2002. Activation of Notch-1 signaling maintains the neoplastic phenotype in human Ras-transformed cells. Nat. Med. 8: 979-986. 


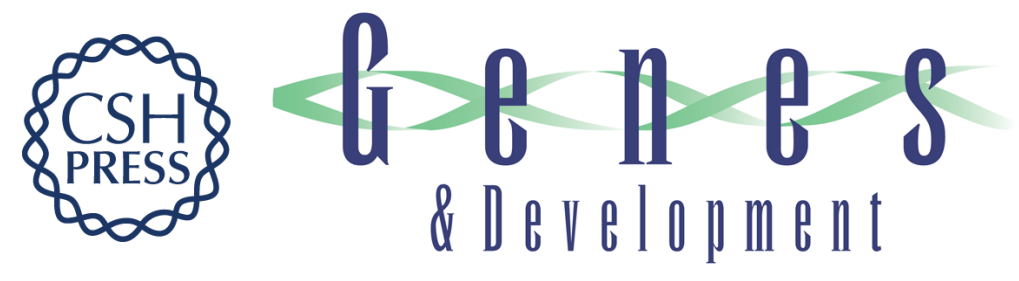

\section{Fishing for the origins of cancer}

Glenn Merlino and Chand Khanna

Genes Dev. 2007, 21:

Access the most recent version at doi:10.1101/gad.1563707

Related Content Effects of RAS on the genesis of embryonal rhabdomyosarcoma

David M. Langenau, Matthew D. Keefe, Narie Y. Storer, et al.

Genes Dev. June , 2007 21: 1382-1395

References This article cites 37 articles, 12 of which can be accessed free at:

http://genesdev.cshlp.org/content/21/11/1275.full.html\#ref-list-1

Articles cited in:

http://genesdev.cshlp.org/content/21/11/1275.full.html\#related-urls

\section{License}

Email Alerting

Receive free email alerts when new articles cite this article - sign up in the box at the top

Service

right corner of the article or click here.

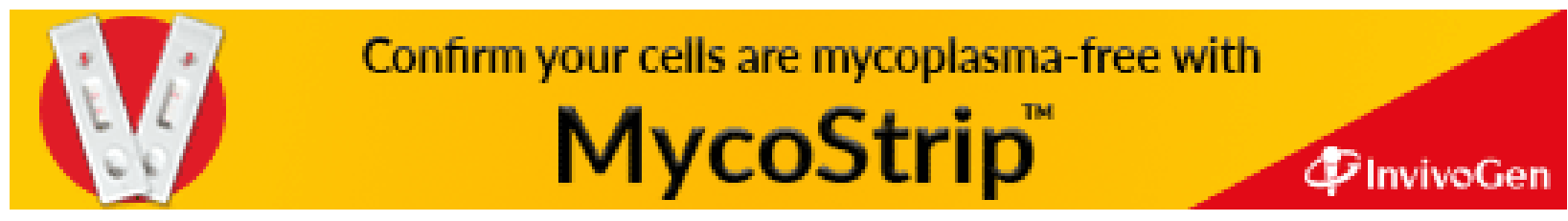

